OPEN ACCESS

Edited by:

Michael Sattler,

Technical University of Munich,

Germany

Reviewed by:

Ann B. Moser

Kennedy Krieger Institute,

United States

Gérard Lizard,

Université de Bourgogne, France

${ }^{*}$ Correspondence:

Stephan Kemp

s.kemp@amsterdamumc.n

Specialty section:

This article was submitted to

Membrane Traffic,

a section of the journal

Frontiers in Cell and Developmental

Biology

Received: 19 May 2020 Accepted: 07 July 2020

Published: 29 July 2020

Citation:

Jaspers YRJ, Ferdinandusse $S$,

Dijkstra IME, Barendsen RW, van Lenthe H, Kulik W, Engelen M, Goorden SMI, Vaz FM and Kemp S (2020) Comparison of the Diagnostic

Performance

of C26:0-Lysophosphatidylcholine and Very Long-Chain Fatty Acids Analysis for Peroxisomal Disorders.

Front. Cell Dev. Biol. 8:690.

doi: $10.3389 /$ fcell.2020.00690

\section{Comparison of the Diagnostic Performance of C26:0-Lysophosphatidylcholine and Very Long-Chain Fatty Acids Analysis for Peroxisomal Disorders}

\author{
Yorrick R. J. Jaspers' ${ }^{1}$, Sacha Ferdinandusse ${ }^{1}$, Inge M. E. Dijkstra', \\ Rinse Willem Barendsen', Henk van Lenthe', Wim Kulik', Marc Engelen², \\ Susan M. I. Goorden ${ }^{1}$, Frédéric M. Vaz' and Stephan Kemp ${ }^{1,2 *}$
}

\begin{abstract}
'Department of Clinical Chemistry, Laboratory Genetic Metabolic Diseases, Amsterdam UMC, Amsterdam Gastroenterology and Metabolism, University of Amsterdam, Amsterdam, Netherlands, ${ }^{2}$ Department of Pediatric Neurology, Amsterdam UMC, Amsterdam Leukodystrophy Center, Emma Children's Hospital, Amsterdam Neuroscience, University of Amsterdam, Amsterdam, Netherlands
\end{abstract}

Peroxisomes are subcellular organelles that are involved in various important physiological processes such as the oxidation of fatty acids and the biosynthesis of bile acids and plasmalogens. The gold standard in the diagnostic work-up for patients with peroxisomal disorders is the analysis of very long-chain fatty acid (VLCFA) levels in plasma. Alternatively, C26:0-lysophosphatidylcholine (C26:0-LPC) can be measured in dried blood spots (DBS) using liquid chromatography tandem mass spectrometry (LC-MS/MS); a fast and easy method but not yet widely used. Currently, little is known about the correlation of C26:0-LPC in DBS and C26:0-LPC in plasma, and how C26:0-LPC analysis compares to VLCFA analysis in diagnostic performance. We investigated the correlation between C26:0-LPC levels measured in DBS and plasma prepared from the same blood sample. For this analysis we included 43 controls and 38 adrenoleukodystrophy (ALD) (21 males and 17 females) and 33 Zellweger spectrum disorder (ZSD) patients. In combined control and patient samples there was a strong positive correlation between DBS C26:0-LPC and plasma C26:0-LPC, with a Spearman's rank correlation coefficient of $r(114)=0.962, p<0.001$. These data show that both plasma and DBS are suitable to determine blood C26:0-LPC levels and that there is a strong correlation between C26:0-LPC levels in both matrices. Following this, we investigated how VLCFA and C26:0-LPC analysis compare in diagnostic performance for 67 controls, 26 ALD males, 19 ALD females, and 35 ZSD patients. For C26:0-LPC, all ALD and ZSD samples had C26:0-LPC levels above the upper limit of the reference range. For C26:0, one out of 67 controls had C26:0 levels above the upper reference range. For 1 out of 26 (1/26) ALD males, 1/19 ALD females and 3/35 ZSD patients, the C26:0 concentration was within the reference range. The C26:0/C22:0 ratio was within the reference range for 0/26 ALD males, 1/19 ALD females and 2/35 


\begin{abstract}
ZSD patients. Overall, these data demonstrate that C26:0-LPC analysis has a superior diagnostic performance compared to VLCFA analysis (C26:0 and C26:0/C22:0 ratio) in all patient groups. Based on our results we recommend implementation of C26:0-LPC analysis in DBS and/or plasma in the diagnostic work-up for peroxisomal disorders.
\end{abstract}

Keywords: adrenoleukodystrophy, peroxisomes, dried bloodspots, C26:0-lysophosphatidylcholine, very longchain fatty acids, VLCFA, beta-oxidation

\section{INTRODUCTION}

Peroxisomes are organelles that are involved in various important physiological processes such as the oxidation of fatty acids and the biosynthesis of bile acids and plasmalogens (Wanders et al., 2010). Peroxisomal disorders affect 1 in 5.000 individuals (Waterham et al., 2016). These disorders can be divided into two subgroups: peroxisome biogenesis disorders and disorders caused by a single peroxisomal enzyme deficiency (Klouwer et al., 2016). Peroxisome biogenesis disorders result from a faulty assembly of peroxisomes and include Zellweger spectrum disorders (ZSD), peroxisomal fission defects, and rhizomelic chondrodysplasia punctata (RCDP) type 1 and 5.

Zellweger spectrum disorder are autosomal recessive disorders caused by mutations in one of 13 PEX genes. ZSD are characterized by a large variety in clinical presentation (Klouwer et al., 2015). Symptoms can include neurological dysfunctions, adrenal insufficiency, and hearing and vision problems. Peroxisomal single enzyme deficiencies include among others, acyl-CoA oxidase deficiency (Ferdinandusse et al., 2007), ACBD5 deficiency (Ferdinandusse et al., 2017), and the most common peroxisomal disorder, adrenoleukodystrophy (ALD) (Moser et al., 2001). Many of these disorders are characterized by the accumulation of very long-chain fatty acids (VLCFAs; $\geq$ C22:0). ALD is the result of a defect in the ABCD1 gene (Mosser et al., 1993) and is characterized by a highly unpredictable clinical manifestation (Kemp et al., 2016). The ABCD1 gene encodes for a peroxisomal membrane protein, referred to as ALDP, that is involved in the transport of VLCFAs into the peroxisome, where they are broken down via $\beta$-oxidation (Singh et al., 1984). A non-functional ALDP results in accumulation of VLCFAs in body fluids and tissues (Moser et al., 1981).

Analysis of VLCFA levels is the gold standard in the diagnostic work-up for peroxisomal disorders. The most commonly used methods are based on gas chromatography (GC), which includes the method developed by Moser et al. (1981) and its improved version in 1991 (Moser and Moser, 1991). Alternatively, methods based on stable isotope dilution using GC combined with mass spectrometry (GCMS) are also widely used (Vreken et al., 1998). While these methods are highly reproducible, they are timeconsuming and labor intensive. Sample preparation can take up to 2 days while analysis takes up to $30 \mathrm{~min}$ per sample using GCMS. Although VLCFAs are regarded as the most important biomarkers for most peroxisomal disorders, false negative results have been reported in approximately $15-20 \%$ of women with ALD (Moser et al., 1999). These factors have led to the development of an alternative diagnostic test: the analysis of C26:0-lysophosphatidylcholine (C26:0-LPC) in dried bloodspots
(DBS) using liquid chromatography tandem mass spectrometry (LC-MS/MS) or flow injection analysis mass spectrometry (FIAMS) (Hubbard et al., 2006; Turgeon et al., 2015; Haynes and Jesús, 2016). C26:0-LPC is elevated in bloodspots from patients with impaired VLCFA metabolism, including women with ALD with normal plasma VLCFA levels (Huffnagel et al., 2017). Importantly, its analysis is considerably faster when compared to VLCFA analysis (Hubbard et al., 2009; Huffnagel et al., 2017; Klouwer et al., 2017). In fact, the identification of C26:0LPC as a specific and sensitive biomarker in bloodspots was of paramount importance for the initiation of ALD newborn screening (Turgeon et al., 2015; Moser et al., 2016; Barendsen et al., 2020).

In the current diagnostic landscape C26:0-LPC in DBS is used for ALD newborn screening. However, the gold standard in the diagnostic work-up for patients with peroxisomal disorders is the analysis of plasma VLCFA levels. The objectives of our study were: (1) To investigate the correlation of C26:0-LPC in DBS and C26:0-LPC in plasma, and (2) To compare the diagnostic performance of C26:0-LPC analysis and VLCFA analysis. We discuss the important factors in the consideration of these methods and provide an overview of possible discrepancies.

\section{MATERIALS AND METHODS}

\section{Patient Samples}

All routine measurements of C26:0-LPC and VLCFA performed at the Laboratory Genetic Metabolic Diseases in the Amsterdam UMC between June 2018 and June 2019 were collected and used for this study. This set was expanded with measurements in additional ALD and ZSD samples. This resulted in samples from 67 controls, 26 ALD males, 19 ALD females and 35 ZSD patients. All ALD patients had confirmed $A B C D 1$ mutations, 32 ZSD patients had confirmed $P E X 1$ mutations, 2 ZSD patients had confirmed PEX6 mutations and 1 ZSD patient had a confirmed PEX26 mutation. Control samples consisted of samples that were screened for a peroxisomal disorder and resulted in a negative outcome. All samples were collected according to the institutional guidelines for blood sampling.

\section{Sample Preparation C26:0-LPC}

Analysis of C26:0-LPC was performed as described earlier by Van de Beek et al. (2016). Briefly, for DBS, a single punch of a $6.2 \mathrm{~mm}(1 / 4 \mathrm{inch})$ bloodspot was extracted with $10 \mu \mathrm{L}$ of an internal standard solution containing $1 \mu \mathrm{mol} / \mathrm{L} \mathrm{D}_{4}$-C26:0lysoPC in $0.5 \mathrm{~mL}$ of methanol by ultrasonication for $5 \mathrm{~min}$ in a sonicator bath (Branson 3510) at room temperature. For plasma, 
$10 \mu \mathrm{L}$ was extracted with $10 \mu \mathrm{L}$ of an internal standard solution containing $1 \mu \mathrm{mol} / \mathrm{L} \mathrm{D}_{4}$-C26:0-lysoPC in $0.5 \mathrm{~mL}$ of acetonitrile by ultrasonication for $5 \mathrm{~min}$ in a sonicator bath (Branson 3510) at room temperature. After centrifugation ( $5 \mathrm{~min}, 14000 \mathrm{RPM}$ ) the resulting methanol (DBS) or acetonitrile (plasma) layer was transferred to a new glass tube and evaporated under a constant stream of nitrogen at $40^{\circ} \mathrm{C}$. The samples were then reconstituted in $50 \mu \mathrm{L}$ methanol, transferred to a sample vial, and capped.

\section{HPLC-MS/MS Analysis}

Samples were injected using an ACQUITY UPLC system (Waters, Milford, MA, United States) on a $50 \times 2.1 \mathrm{~mm}$, 2.6 $\mathrm{mm}$ particle diameter Kinetex C8 column (Phenomenex, Torrance, CA, United States). The column was held at a constant temperature of $50^{\circ} \mathrm{C}$. The composition of mobile phase A was $0.1 \%$ formic acid in water and mobile phase B was $0.1 \%$ formic acid in methanol. The gradient used was as follows: $T=0 \mathrm{~min}$ : $36 \% \mathrm{~A}, 64 \% \mathrm{~B}$, flow $0.4 \mathrm{~mL} / \mathrm{min}$ toward $T=6 \mathrm{~min}: 0 \% \mathrm{~A}$, $100 \% \mathrm{~B}$, flow $0.4 \mathrm{~mL} / \mathrm{min} ; T=6-11 \mathrm{~min}: 0 \% \mathrm{~A}, 100 \% \mathrm{~B}$, flow $0.4 \mathrm{~mL} / \mathrm{min}$, and $T=11-11.1$ back to $36 \% \mathrm{~A}, 64 \% \mathrm{~B}$, flow $0.4 \mathrm{~mL} / \mathrm{min}$. Detection was done using a Quattro Premier XE (Waters, Milford, MA, United States) using electrospray ionization in positive mode. The source temperature was $130^{\circ} \mathrm{C}$, and capillary voltage was $3.5 \mathrm{kV}$. Multiple reaction monitoring $(\mathrm{MRM})$ was done on masses $636.50>104.10$ and $640.50>104.10$ with a dwell time of $0.03 \mathrm{~s}$. Argon was used as a collision gas.

\section{Sample Preparation VLCFAs}

Very long-chain fatty acids analysis was performed essentially as described earlier by Vreken et al. In a glass tube, $100 \mu \mathrm{L}$ plasma was added to $100 \mu \mathrm{L}$ internal standard solution dissolved in toluene that consisted of $50 \mu \mathrm{mol} / \mathrm{L} \mathrm{D}_{4}$-C22:0, $50 \mu \mathrm{mol} / \mathrm{L} \mathrm{D}_{4}$ C24:0, $1 \mu \mathrm{mol} / \mathrm{L} \mathrm{D}_{4}$-C26:0. Acidic hydrolysis was performed by adding $2 \mathrm{~mL}$ of a $0.5 \mathrm{~mol} / \mathrm{L} \mathrm{HCl}$ in acetonitrile and incubating at $110^{\circ} \mathrm{C}$ for $45 \mathrm{~min}$. After cooling to room temperature, fatty acids were extracted into $4 \mathrm{~mL}$ of hexane. The resulting hexane layer was transferred to a new glass tube and washed with $3.5 \mathrm{~mL} 1 \mathrm{M}$ $\mathrm{KOH}$. Next, the hexane layer was removed and $600 \mu \mathrm{L} 25 \% \mathrm{HCl}$ was added. Fatty acids were extracted with $4 \mathrm{~mL}$ of hexane. The hexane layer was then transferred to a new glass tube and dried under nitrogen at $50^{\circ} \mathrm{C}$. Fatty acids were derivatized with $50 \mu \mathrm{L}$ pyridine and $50 \mu \mathrm{L}$ MTBSTFA at $80^{\circ} \mathrm{C}$ for $30 \mathrm{~min}$. The solvent was then evaporated under nitrogen at $50^{\circ} \mathrm{C}$ and the derivatized fatty acids were reconstituted in $200 \mu \mathrm{L}$ hexane and transferred to autosampler vials.

\section{GCMS Analysis}

Analysis was performed on an Agilent 7890B GC using a 5977A MSD mass spectrometer for compound detection. Separation was achieved on a Cp-sil 19, $25 \mathrm{~m}^{*} 0.25 \mathrm{~mm}^{*} 0.20 \mu \mathrm{m}$ column. Temperature gradient was as follows: $60^{\circ} \mathrm{C}$ was held for $1.5 \mathrm{~min}$ after which the temperature increased to $240^{\circ} \mathrm{C}$ at a rate of $10^{\circ} \mathrm{C} / \mathrm{min}$. The temperature was then increased to 270 , at $4^{\circ} \mathrm{C} / \mathrm{min}$. Finally the temperature increased to $300^{\circ} \mathrm{C}$ at $20^{\circ} \mathrm{C} / \mathrm{min}$. An injection volume of $1 \mu \mathrm{L}$ was used in splitless mode using a splitless time of $1.5 \mathrm{~min}$. The carrier gas was
Helium $(50 \mathrm{kPa})$. Single ion monitoring mode was used for detection of VLCFAs.

\section{RESULTS}

\section{C26:0-LPC in DBS Versus Plasma}

To investigate the correlation between C26:0-LPC in DBS and C26:0-LPC in plasma we measured C26:0-LPC in DBS and plasma prepared from the same blood sample. For this analysis we included 43 control, 21 ALD males, 17 ALD females and 33 ZSD samples (Figure 1). The median C26:0LPC level in DBS of controls was $0.033 \mu \mathrm{mol} / \mathrm{L}$ (range $0.016-$ $0.063 \mu \mathrm{mol} / \mathrm{L}$ ), in ALD males it was $0.425 \mu \mathrm{mol} / \mathrm{L}$ (range 0.224 $1.208 \mu \mathrm{mol} / \mathrm{L}$ ), in ALD females it was $0.276 \mu \mathrm{mol} / \mathrm{L}$ (range $0.080-0.497 \mu \mathrm{mol} / \mathrm{L}$ ) and in ZSD patients it was $0.470 \mu \mathrm{mol} / \mathrm{L}$ (range $0.124-2.881 \mu \mathrm{mol} / \mathrm{L}$ ). The upper limit of the reference range in our laboratory is $0.072 \mu \mathrm{mol} / \mathrm{L}$. All patients had elevated levels of C26:0-LPC. There was a clear separation between controls and patients; the highest control level was $0.063 \mu \mathrm{mol} / \mathrm{L}$ and the lowest patient value was $0.080 \mu \mathrm{mol} / \mathrm{L}$ in a sample from an ALD female. For C26:0-LPC in plasma, the median level in controls was $0.040 \mu \mathrm{mol} / \mathrm{L}$ (range $0.011-$ $0.063 \mu \mathrm{mol} / \mathrm{L}$ ), in ALD males it was $0.333 \mu \mathrm{mol} / \mathrm{L}$ (range $0.127-0.736 \mu \mathrm{mol} / \mathrm{L})$, in ALD females it was $0.266 \mu \mathrm{mol} / \mathrm{L}$ (range $0.118-0.576 \mu \mathrm{mol} / \mathrm{L}$ ) and in ZSD it was $0.445 \mu \mathrm{mol} / \mathrm{L}$ (range $0.074-2.485 \mu \mathrm{mol} / \mathrm{L}$ ). All patients had elevated levels of C26:0-LPC. The highest control level was $0.063 \mu \mathrm{mol} / \mathrm{L}$ and

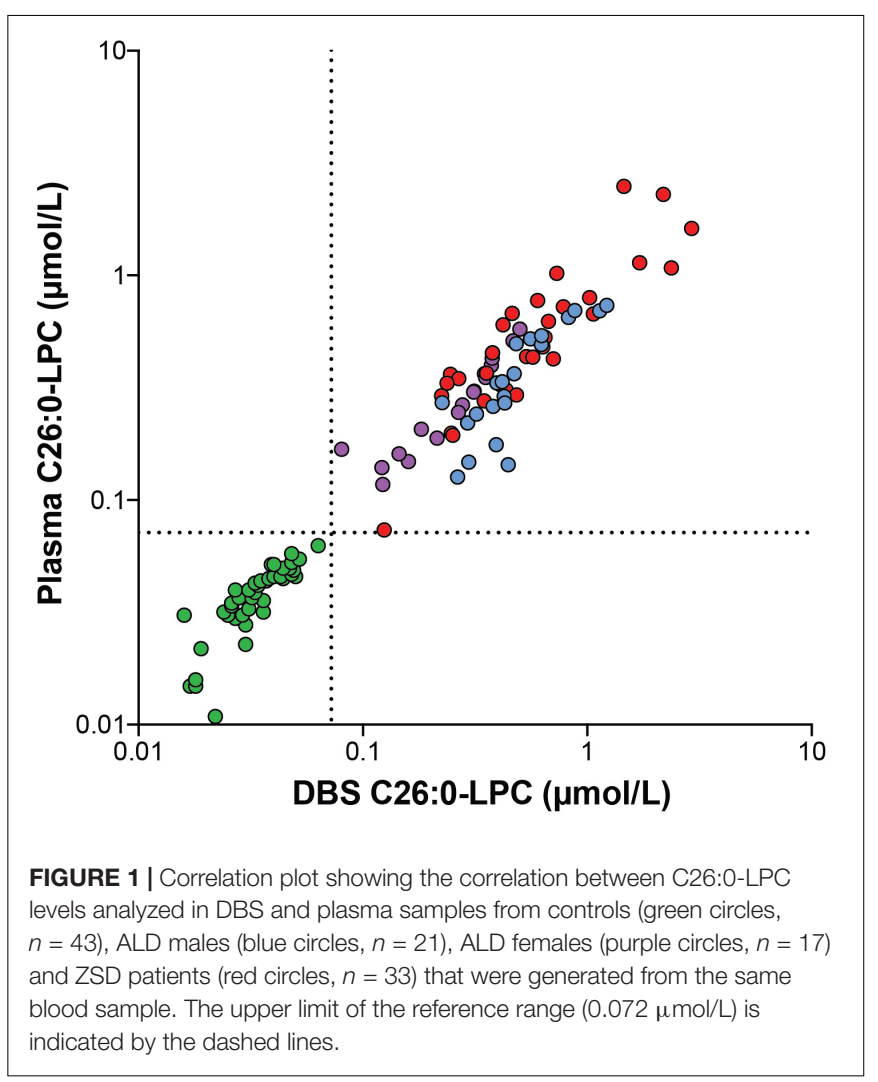


the lowest patient level was $0.074 \mu \mathrm{mol} / \mathrm{L}$ in a sample from a ZSD patient. We investigated the correlation between C26:0LPC levels measured in DBS and C26:0-LPC levels measured in plasma with a Spearman's rank-order correlation. In the combined set of both control and patient samples there was a very strong positive correlation between DBS C26:0-LPC and plasma C26:0-LPC with a Spearman's rank correlation coefficient of $r(114)=0.962, p<0.001$. For controls alone, it was $r(43)=0.907, p<0.001$. For patients alone, it was $r(71)=0.861, p<0.001$. These data show that both plasma and DBS are suitable to determine blood C26:0-LPC levels and that there is a strong correlation between C26:0-LPC levels in both matrices. The strong correlation between plasma and DBS allowed for combining plasma and DBS C26:0-LPC data for comparison to VLCFAs as a diagnostic marker for peroxisomal disorders.

\section{C26:0-LPC Levels vs. VLCFA}

To compare the diagnostic performance of plasma VLCFA analysis (C26:0 concentration and $\mathrm{C} 26: 0 / \mathrm{C} 22: 0$ ratio) and $\mathrm{C} 26: 0$ LPC analysis in plasma and DBS, we measured these metabolites in samples from controls $(n=67)$, ALD males $(n=26)$, ALD females $(n=19)$ and ZSD patients $(n=35)$ (Figure 2). For C26:0, the median level in controls was $0.67 \mu \mathrm{mol} / \mathrm{L}$ (range $0.37-1.34 \mu \mathrm{mol} / \mathrm{L}$ ), in ALD males it was $2.92 \mu \mathrm{mol} / \mathrm{L}$ (range: 1.19-5.01 $\mu \mathrm{mol} / \mathrm{L}$ ), in ALD females it was $1.81 \mu \mathrm{mol} / \mathrm{L}$ (range $1.11-4.06 \mu \mathrm{mol} / \mathrm{L})$ and in ZSD patients it was $2.41 \mu \mathrm{mol} / \mathrm{L}(0.95-$ $9.74 \mu \mathrm{mol} / \mathrm{L})$. The upper limit of the reference range in our laboratory for C26:0 in plasma is $1.32 \mu \mathrm{mol} / \mathrm{L}$. One out of 67 controls had C26:0 levels above the reference range. One out of 26 ALD males, 1/19 ALD females and 3/35 ZSD patients had a C26:0 concentration in the reference range. For the C26:0/C22:0 ratio, the median ratio in controls was 0.012 (range $0.008-$ 0.053), in ALD males it was 0.055 (range: 0.033-0.09), in ALD females it was $0.03(0.02-0.05)$ and in ZSD patients it was 0.05 (range: 0.02-0.39). The upper limit of the reference range in our laboratory for the C26:0/C22:0 ratio in plasma is 0.02 . Six out of 67 controls had a C26:0/C22:0 ratio above the upper limit of the reference range. The elevated $\mathrm{C} 26: 0 / \mathrm{C} 22: 0$ ratio of these samples was the result of relatively low $\mathrm{C} 22$ concentration [range: 12$59 \mu \mathrm{mol} / \mathrm{L}$ (reference range: $40-119 \mu \mathrm{mol} / \mathrm{L}$ )] in combination with normal C26 concentration (range: 0.61-1.29 $\mu \mathrm{mol} / \mathrm{L}$ ). A peroxisomal disorder diagnosis was rejected in these six control individuals because all other peroxisomal parameters

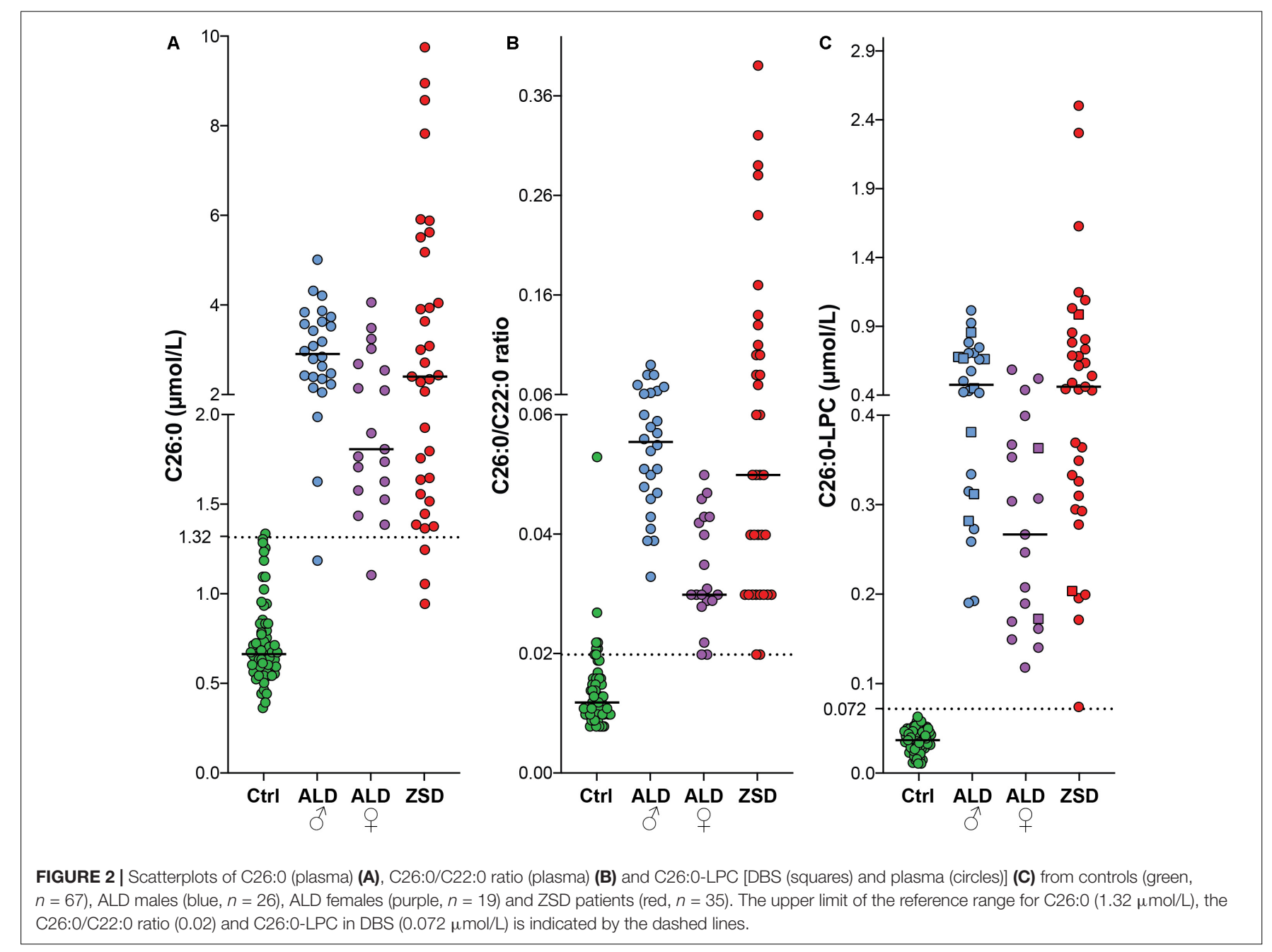


(phytanic acid, bile acid intermediates, and plasmalogens) were normal, and VLCFA analysis in a repeat sample showed no abnormalities. None of the ALD males, 1/19 ALD females and 2/35 ZSD patients had a C26:0/C22:0 ratio within the reference range. For C26:0-LPC measurements in DBS and plasma, the median level in controls was $0.037 \mu \mathrm{mol} / \mathrm{L}$ (range: $0.011-$ $0.063 \mu \mathrm{mol} / \mathrm{L}$ ), in ALD males it was $0.467 \mu \mathrm{mol} / \mathrm{L}$ (range: $0.190-$ $1.004 \mu \mathrm{mol} / \mathrm{L}$ ), in ALD females it was $0.266 \mu \mathrm{mol} / \mathrm{L}$ (range: $0.118-0.576 \mu \mathrm{mol} / \mathrm{L}$ ) and in ZSD patients it was $0.453 \mu \mathrm{mol} / \mathrm{L}$ (range: $0.074-2.485 \mu \mathrm{mol} / \mathrm{L}$ ). The upper limit of the reference range in our laboratory is $0.072 \mu \mathrm{mol} / \mathrm{L}$. All controls had a C26:0LPC concentration below this upper limit and all patients had C26:0-LPC levels above the upper limit of the reference range. In this cohort, only C26:0-LPC showed a complete separation between controls and patients.

Since the C26:0-LPC and VLCFA analyses were performed on samples generated from the same blood sample, we investigated the correlation between the 3 diagnostic markers with a Spearman's rank-order correlation (Figure 3). When, combining controls and patients there was a strong positive correlation between all 3 biomarkers. For the C26:0/C22:0 ratio and C26:0 concentration the Spearman's rank correlation coefficient was $r$ $(147)=0.892, p<0.001$; for C26:0-LPC and C26:0 levels it was $r$ $(147)=0.892, p<0.001$; and for C26:0-LPC and the C26:0/C22:0 ratio it was $r(147)=0.816, p<0.001$.

\section{DISCUSSION}

\section{Comparison of C26:0-LPC Levels in DBS and Plasma}

The first objective of this study was to investigate the correlation between C26:0-LPC levels measured in DBS and plasma. To this end, we compared C26:0-LPC in DBS and plasma samples that were derived from the same blood sample. Our results demonstrate that both in control and patient samples C26:0LPC levels in DBS and in plasma are very strongly correlated $(r(114)=0.962, p<0.001)$. C26:0-LPC in DBS is an effective biomarker for ALD and ZSD patients (Hubbard et al., 2006, 2009; Huffnagel et al., 2017; Klouwer et al., 2017). In some cases, DBS sampling provides considerable advantages over plasma. DBS sampling is relatively non-invasive compared to whole blood sampling. It can be performed in a patient's home or at a local point of care, and requires less personnel training (McDade et al., 2007). DBS can be easily shipped by regular mail which is much cheaper and less logistically challenging than transporting plasma or whole blood, which requires specific supply chain conditions by courier, such as dry ice for plasma or controlled temperature for whole blood. Despite these advantages DBS do have diagnostic limitations compared to plasma. For a complete diagnostic testing for peroxisomal disorders not only VLCFAs analysis is required, but also phytanic acid, pristanic acid, di- and trihydroxycholestanoic acid analysis in plasma and plasmalogen analysis in erythrocytes. Because currently there is no evidence that these tests can be done in DBS, plasma is still needed in the diagnostic work-up for patients with peroxisomal disorders.

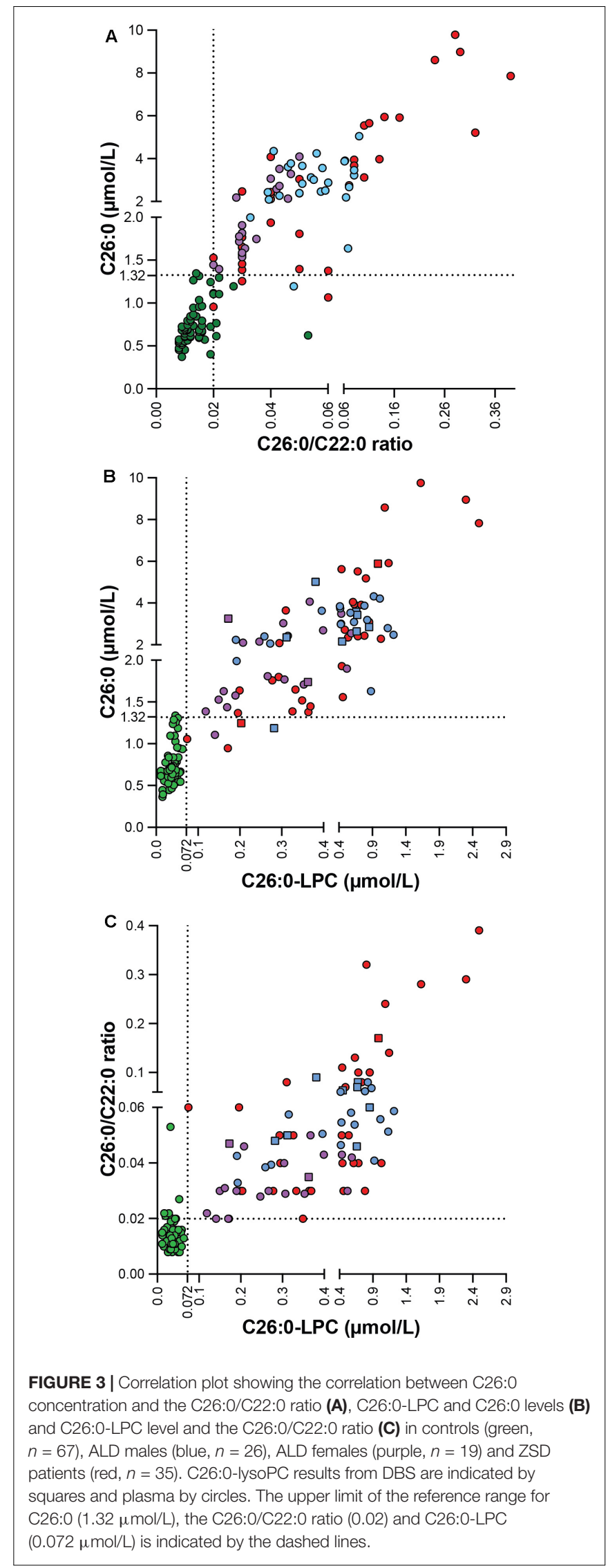




\section{Comparison of C26:0-LPC and VLCFA}

Traditionally, VLCFA analysis plays an important role in the diagnosis of peroxisomal disorders. However, the analysis of VLCFAs using GCMS is time-consuming and labor intensive. C26:0-LPC is a potential alternative marker for VLCFAs. Therefore, the second objective of this study was to compare the diagnostic performance of VLCFA and C26:0-LPC analysis and systematically evaluate potential differences in outcome between these markers. To this end, we measured VLCFA levels and C26:0-LPC in samples from controls, ALD patients (males and females) and ZSD patients. Almost all patient samples showed increased C26:0/C22:0 ratios. None of the ALD males, 1/19 ALD females and 2/35 ZSD patients had a C26:0/C22:0 ratio within the reference range. The finding that 6 samples from the control group fell outside the reference range of C26:0/C22:0 indicates that false positives should be considered. For these samples the false positive results were due to low C22:0 and normal C26:0 concentrations. For this reason, plasma VLCFA analysis always includes both the analysis of $\mathrm{C} 26: 0$ and the $\mathrm{C} 26: 0 / \mathrm{C} 22: 0$ ratio as a means of improving the accuracy of the readout. The combination of these 2 markers increases the sensitivity of the analysis. Furthermore, false-positive results in VLCFA levels have been reported in individuals without a peroxisomal defect. For example, diabetic ketoacidosis, a ketogenic diet or hemolysis of the blood sample can result in false-positive VLCFA levels (Kishimoto et al., 1980; Brown et al., 1982; Theda et al., 1993). Importantly, non-fasted blood samples obtained from individuals who had consumed peanut butter in the hours prior to blood collection have VLCFA levels that are in the abnormal range (Lam et al., 2012). This is most likely due to direct dietary effects on plasma components that contain VLCFA, including lipoprotein particles and cholesterol esters (Engelen et al., 2010; Wanders et al., 2010). This dietary influence on C26:0 levels is minimized in practice by blood sampling in a fasted state. However, fasting is a considerable hindrance for the patient and non-compliance might occur. There is currently no evidence that C26:0-LPC levels are affected by diet. In blood, C26:0-LPC is primarily found as a membrane component in cells such as erythrocytes (Tanaka et al., 1989). Since erythrocytes cycle for 2 to 4 months, the C26:0LPC level is expected to be relatively stable over this period with little effect of dietary influences (Cohen et al., 2008; Mock et al., 2011). This simplifies sampling procedures and results in a lower number of false positives. Indeed, all C26:0-LPC levels measured in control samples in this study were within reference range. All ALD and ZSD samples had increased C26:0-LPC levels. Overall our data show that C26:0-LPC measurement has a superior diagnostic performance compared to the traditional VLCFA analysis in all patient groups and results in less false positives. Consistent with earlier work, males tended to have higher C26:0 and C26:0/C22:0 levels than females (O'Neill et al., 1984; Moser et al., 1999; Engelen et al., 2014), which was also observed for C26:0-LPC. It has been well-established that plasma VLCFA analysis may result in a false negative result in approximately $15-20 \%$ of women with ALD (Moser et al., 1999). The results of this study are in agreement with our earlier work showing that women with ALD have elevated C26:0-LPC, including those with normal plasma C26:0 and C26:0/C22:0 (Huffnagel et al.,
2017, 2019). Taken together these results show that the analysis of C26:0-LPC, either in DBS or in plasma, considerably increases the sensitivity of detecting disorders associated with a defect in peroxisomal beta oxidation. Albeit, some caution is warranted with the interpretation of a normal C26:0-LPC result given the fact that the lowest level measured in a DBS sample from an ALD female $(0.080 \mu \mathrm{mol} / \mathrm{L})$ and a plasma sample from a ZSD patient $(0.074 \mu \mathrm{mol} / \mathrm{L})$ were very close to the upper limit of the reference range $(0.072 \mu \mathrm{mol} / \mathrm{L})$ (Figure 1). In line with plasma VLCFA levels, C26:0-LPC levels in ALD males and females overlap with the levels measured in ZSD patients. No differentiation can be made between ALD patients and ZSD patients based on either C26:0, C26:0/C22:0 or C26:0-LPC level alone. Analysis of other metabolites and genetic analysis are required to differentiate between these disorders and confirm the diagnosis. Besides a superior diagnostic performance, the LCMS-based analytical method for C26:0-LPC is far less time consuming and labor intensive than the GCMS-based VLCFA analysis. Increased C26:0 levels have been reported in peripheral blood from patients with Alzheimer's disease and vascular dementia (Lizard et al., 2012; Zarrouk et al., 2015). It would be interesting to evaluate C26:0-LPC in these patients.

In conclusion, this study demonstrates that C26:0-LPC analysis performed in DBS and in plasma are strongly correlated. Furthermore, our results demonstrate the analysis of C26:0-LPC has superior diagnostic performance for ALD and ZSD patients compared to C26:0 and C26:/C22:0. Based on our results we recommend implementation of C26:0-LPC analysis in DBS and/or plasma in the diagnostic work-up for peroxisomal disorders.

\section{DATA AVAILABILITY STATEMENT}

The raw data supporting the conclusions of this article will be made available by the authors, without undue reservation.

\section{AUTHOR CONTRIBUTIONS}

YJ, SF, WK, ME, SG, FV, and SK conceived the project. ID and HL performed the VLCFA and C26:0-LPC analyses. YJ and SK performed the data analyses. YJ, SF, RB, ME, SG, FV, and SK wrote the manuscript. All authors contributed to the article and approved the submitted version.

\section{FUNDING}

This work was supported by The Netherlands Organization for Health Research and Development (ZonMw), project number 543002004 to SK.

\section{ACKNOWLEDGMENTS}

The authors thank Martin Vervaart, Elise van der Sluijs, and Henny Rusch for technical assistance and Femke Klouwer for expert assistance. 


\section{REFERENCES}

Barendsen, R. W., Dijkstra, I. M. E., Visser, W. F., Alders, M., Bliek, J., Boelen, A., et al. (2020). Adrenoleukodystrophy newborn screening in the Netherlands (SCAN study): the X-factor. Front. Cell Dev. Biol. 8:499. doi: 10.3389/fcell.2020. 00499

Brown, F. R., Duyn, M. A. S., van Moser, A. B., Schulman, J. D., Rizzo, W. B., Snyder, R. D., et al. (1982). Adrenoleukodystrophy: effects of dietary restriction of very long chain fatty acids and of administration of carnitine and clofibrate on clinical status and plasma fatty acids. Johns Hopkins Med. J. 151, 164-172.

Cohen, R. M., Franco, R. S., Khera, P. K., Smith, E. P., Lindsell, C. J., Ciraolo, P. J., et al. (2008). Red cell life span heterogeneity in hematologically normal people is sufficient to alter HbAlc. Blood 112, 4284-4291. doi: 10.1182/blood-200804- 154112

Engelen, M., Barbier, M., Dijkstra, I. M. E., Schür, R., Bie, R. M. A., De Verhamme, C., et al. (2014). X-linked adrenoleukodystrophy in women: a cross-sectional cohort study. Brain 137, 693-706. doi: 10.1093/brain/awt361

Engelen, M., Ofman, R., Dijkgraaf, M. G. W., Hijzen, M., Wardt, L. A., Van Der Geel, B. M., et al. (2010). Lovastatin in X-linked adrenoleukodystrophy. N. Engl. J. Med. 362, 276-277.

Ferdinandusse, S., Denis, S., Hogenhout, E. M., Koster, J., Roermund, C. W. T., van IJlst, L., et al. (2007). Clinical, biochemical, and mutational spectrum of peroxisomal acyl-coenzyme A oxidase deficiency. Hum. Mutat. 28, 904-912. doi: 10.1002/humu.20535

Ferdinandusse, S., Falkenberg, K. D., Koster, J., Mooyer, P. A., Jones, R., Roermund, C. W. T., et al. (2017). ACBD5 deficiency causes a defect in peroxisomal very long-chain fatty acid metabolism. J. Med. Genet. 54, 330-337. doi: 10.1136/ jmedgenet-2016-104132

Haynes, C. A., and Jesús, V. R. D. (2016). Simultaneous quantitation of hexacosanoyl lysophosphatidylcholine, amino acids, acylcarnitines, and succinylacetone during FIA-ESI-MS/MS analysis of dried blood spot extracts for newborn screening. Clin. Biochem. 49, 161-165. doi: 10.1016/j.clinbiochem. 2015.09.011

Hubbard, W. C., Moser, A. B., Liu, A. C., Jones, R. O., Steinberg, S. J., Lorey, F., et al. (2009). Newborn screening for X-linked adrenoleukodystrophy (X-ALD): validation of a combined liquid chromatography-tandem mass spectrometric (LC-MS/MS) method. Mol. Genet. Metab. 97, 212-220. doi: 10.1016/j.ymgme. 2009.03.010

Hubbard, W. C., Moser, A. B., Tortorelli, S., Liu, A., Jones, D., and Moser, H. (2006). Combined liquid chromatography-Tandem mass spectrometry as an antytical method for high throughput screening for X-linked adrenoleukodystrophy and other peroxisomal disorders: preliminary findings. Mol. Genet. Metab. 89, 185-187. doi: 10.1016/j.ymgme.2006.05.001

Huffnagel, I. C., Dijkgraaf, M. G. W., Janssens, G. E., Weeghel, M., Van Geel, B. M., Van Poll-The, B. T., et al. (2019). Disease progression in women with X-linked adrenoleukodystrophy is slow. Orphanet J. Rare Dis. 14, 1-15.

Huffnagel, I. C., van de Beek, M. C., Showers, A. L., Orsini, J. J., Klouwer, F. C. C., Dijkstra, I. M. E., et al. (2017). Comparison of C26:0-carnitine and C26:0-lysophosphatidylcholine as diagnostic markers in dried blood spots from newborns and patients with adrenoleukodystrophy. Mol. Genet. Metab. 122, 209-215. doi: 10.1016/j.ymgme.2017.10.012

Kemp, S., Huffnagel, I. C., Linthorst, G. E., Wanders, R. J., and Engelen, M. (2016). Adrenoleukodystrophy - Neuroendocrine pathogenesis and redefinition of natural history. Nat. Rev. Endocrinol. 12, 606-615. doi: 10.1038/nrendo.2016.90

Kishimoto, Y., Moser, H. W., Kawamura, N., Platt, M., Pallante, S. L., and Fenselau, C. (1980). Adrenoleukodystrophy: evidence that abnormal very long chain fatty acids of brain cholesterol esters are of exogenous origin. Top. Catal. 96, 69-76. doi: 10.1016/0006-291x(80)91182-1

Klouwer, F. C. C., Berendse, K., Ferdinandusse, S., Wanders, R. J. A., Engelen, M., and Poll-The, B. T. (2015). Zellweger spectrum disorders: clinical overview and management approach Inherited metabolic diseases. Orphanet J. Rare Dis. 10, $1-11$.

Klouwer, F. C. C., Ferdinandusse, S., Lenthe, H., van Kulik, W., Wanders, R. J. A., Poll-The, B. T., et al. (2017). Evaluation of C26:0-lysophosphatidylcholine and C26:0-carnitine as diagnostic markers for Zellweger spectrum disorders. J. Inherit. Metab. Dis. 40, 875-881. doi: 10.1007/s10545-017-0064-0

Klouwer, F. C. C., Huffnagel, I. C., Ferdinandusse, S., Waterham, H. R., Wanders, R. J. A., Engelen, M., et al. (2016). Clinical and biochemical pitfalls in the diagnosis of peroxisomal disorders. Neuropediatrics 47, 205-220. doi: 10.1055/ s- 0036- 1582140

Lam, C., Wong, D., Cederbaum, S., Lim, B., and Qu, Y. (2012). Peanut consumption increases levels of plasma very long chain fatty acids in humans. Mol. Genet. Metab. 107, 620-622. doi: 10.1016/j.ymgme.2012.07.015

Lizard, G., Rouaud, O., Demarquoy, J., Cherkaoui-Malki, M., and Iuliano, L. (2012). Potential roles of peroxisomes in alzheimer's disease and in dementia of the alzheimer's type. J. Alzheimer's Dis. 29, 241-254.

McDade, T. W., Williams, S., and Snodgrass, J. J. (2007). What a drop can do: dried blood spots as a minimally invasive method for integrating biomarkers into population-based research. Demography 44, 899-925. doi: 10.1353/dem. 2007.0038

Mock, D. M., Matthews, N. I., Zhu, S., Strauss, R. G., Schmidt, R. L., Nalbant, D., et al. (2011). Red blood cell (RBC) survival determined in humans using RBCs labeled at multiple biotin densities. Transfusion 51, 1047-1057. doi: 10.1111/j.1537-2995.2010.02926.x

Moser, A. B., Jones, R. O., Hubbard, W. C., Tortorelli, S., Orsini, J. J., Caggana, M., et al. (2016). Newborn screening for X-Linked adrenoleukodystrophy. Int. J. Neonatal. Screen 2:15. doi: 10.3390/ijns204 0015

Moser, A. B., Kreiter, N., Bezman, L., Lu, S., Raymond, G. V., Naidu, S., et al. (1999). Plasma very long chain fatty acids in 3,000 peroxisome disease patients and 29,000 controls. Ann. Neurol. 45, 100-110. doi: 10.1002/1531-8249(199901)45: $1<100:$ :aid-art $16>3.0 . c 0 ; 2-\mathrm{u}$

Moser, H. W., and Moser, A. B. (1991). "Measurement of saturated very long chain fatty acid in plasma," in Techniques in Diagnostic Human Biochemical Genetics, ed. F. A. Hommes (New York, NY: Wiley-Liss).

Moser, H. W., Moser, A. B., Frayer, K. K., Chen, W., Schulman, J. D., O’Neill, B. P., et al. (1981). Adrenoleukodystrophy: increased plasma content of saturated very long chain fatty acids. Neurology 31, 1241-1241. doi: 10.1212/wnl.31.10.1241

Moser, H. W., Smith, K. D., Watkins, P. A., Powers, J., and Moser, A. B. (2001). "X-Linked Adrenoleukodystrophy," in The Metabolic and Molecular Bases of Inherited Disease, eds C. R. Scriver, M. D. Beaudet, W. S. Sly, and D. Vall (New York, NY: McGraw-Hill), 3257-3301.

Mosser, J., Douar, A. M., Sarde, C. O., Kioschis, P., Feil, R., Moser, H., et al. (1993). Putative X-linked adrenoleukodystrophy gene shares unexpected homology with ABC transporters. Nature 361, 726-730. doi: 10.1038/36 $1726 \mathrm{a} 0$

O’Neill, B. P., Moser, H. W., Saxena, K. M., and Marmion, L. C. (1984). Adrenoleukodystrophy: clinical and biochemical manifestations in carriers. Neurology 34, 798-798. doi: 10.1212/wnl.34.6.798

Singh, I., Moser, E., Moser, H. W., and Kishimoto, Y. (1984). Adrenoleukodystrophy: impaired oxidation of very long chain fatty acids in white blood cells, cultured skin fibroblasts,; and amniocytes. Pediatr. Res. 18, 286-290. doi: 10.1203/00006450-19840300000016

Tanaka, K., Shimada, M., Naruto, T., Yamamoto, H., Nishizawa, K., and Saeki, Y. (1989). Very long-chain fatty acids in erythrocyte membrane phospholipids in adrenoleukodystrophy. Pediatr. Int. 31, 136-143. doi: 10.1111/j.1442-200x. 1989.tb01279.x

Theda, C., Woody, R. C., Naidu, S., Moser, A. B., and Moser, H. W. (1993). Increased very long chain fatty acids in patients on a ketogenic diet: a cause of diagnostic confusion. J. Pediatr. 122, 724-726. doi: 10.1016/s0022-3476(06) 80013-2

Turgeon, C. T., Moser, A. B., Mørkrid, L., Magera, M. J., Gavrilov, D. K., Oglesbee, D., et al. (2015). Streamlined determination of lysophosphatidylcholines in dried blood spots for newborn screening of X-linked adrenoleukodystrophy. Mol. Genet. Metab. 114, 46-50. doi: 10.1016/j.ymgme.2014. 11.013

Van de Beek, M. C., Dijkstra, I. M. E., Lenthe, H., Van Ofman, R., GoldhaberPasillas, D., Schauer, N., et al. (2016). C26:0-Carnitine is a new biomarker for X-linked adrenoleukodystrophy in mice and man. PLoS One 11:e0154597. doi: 10.1371/journal.pone.0154597

Vreken, P., Lint, A. E. M., Van Bootsma, A. H., Overmars, H., Wanders, R. J. A., and Gennip, A. H. (1998). Rapid stable isotope dilution analysis of very-long-chain fatty acids, pristanic acid and phytanic acid using gas chromatography-electron impact mass spectrometry. J. Chromatogr. B Biomed. Appl. 713, 281-287. doi: 10.1016/s0378-4347(98)00186-8 
Wanders, R. J. A., Ferdinandusse, S., Brites, P., and Kemp, S. (2010). Peroxisomes, lipid metabolism and lipotoxicity. Biochim. Biophys. Acta Mol. Cell Biol. Lipids 1801, 272-280. doi: 10.1016/j.bbalip.2010.01.001

Waterham, H. R., Ferdinandusse, S., and Wanders, R. J. A. (2016). Human disorders of peroxisome metabolism and biogenesis. Biochim. Biophys. Acta Mol. Cell Res. 1863, 922-933. doi: 10.1016/j.bbamcr.2015. 11.015

Zarrouk, A., Riedinger, J. M., Ahmed, S. H., Hammami, S., Chaabane, W., Debbabi, M., et al. (2015). Fatty acid profiles in demented patients: identification of hexacosanoic acid (C26:0) as a blood lipid biomarker of dementia. J. Alzheimer's Dis. 44, 1349-1359. doi: 10.3233/jad- 142046
Conflict of Interest: The authors declare that the research was conducted in the absence of any commercial or financial relationships that could be construed as a potential conflict of interest.

Copyright $\odot 2020$ Jaspers, Ferdinandusse, Dijkstra, Barendsen, van Lenthe, Kulik, Engelen, Goorden, Vaz and Kemp. This is an open-access article distributed under the terms of the Creative Commons Attribution License (CC BY). The use, distribution or reproduction in other forums is permitted, provided the original author(s) and the copyright owner(s) are credited and that the original publication in this journal is cited, in accordance with accepted academic practice. No use, distribution or reproduction is permitted which does not comply with these terms. 